\title{
甘蓝型油菜不同抗倒性材料中木质素代谢途径关键基因表达特点
}

黄杰恒 ${ }^{* *}$ 李 威 ${ }^{* *}$ 曲存民 刘列钊 徐新福 王 瑞 李加纳 ${ }^{*}$

西南大学农学与生物科技学院 / 南方山地农业教育部工程研究中心, 重庆 400716

摘 要: 植物体内木质素的含量不仅与植物的抗病性有关, 也与植物的抗倒伏性状密切联系。本试验以 15 个抗倒性 等级不同的甘蓝型油菜品种为材料, 分别在初花期和青荚期测定茎秆中部木质素含量, 同时用 qRT-PCR 的方法分析 了木质素代谢途径中 6 个关键基因 $P A L 、 4 C L 、 C 4 H 、 C C R 1 、 C C R 2$ 和 $C A D$ 的表达量差异。结果表明, 油菜茎秆木 质素从初花期到青荚期平均增加了 28\%，抗倒性强的材料木质素增加最明显，达到 $33.5 \%$; 不同时期，不同抗倒伏性 的油菜茎秆中部木质素含量差异均极显著; 各基因表达量在两个时期间差异均显著或极显著, 但在不同抗倒性材料 间，只有基因 $P A L 、 4 C L$ 和 CCR1 表达量间差异显著; 基因 $P A L$ 的表达量与木质素含量相关性最强, 4CL 基因的表达 量与其他关键酶基因表达量相关性最强。综上所述，木质素的合成积累由代谢途径中各个环节关键基因所共同决定， 但基因 $P A L$ 和 $4 C L$ 对木质素的代谢影响更明显。

关键词: 木质素; 苂光定量 PCR; 代谢途径; 抗倒性

\section{Expression Characteristics of Key Genes in Lignin Pathway among Different Lodging Resistance Lines of Brassica napus $\mathbf{L}$.}

HUANG Jie-Heng $^{* *}$, LI Wei** ${ }^{* *}$ QU Cun-Min, LIU Lie-Zhao, XU Xin-Fu, WANG Rui, and LI Jia-Na ${ }^{*}$

Engineering Research Center of South Upland Agriculture / College of Agronomy and Biotechnology, Southwest University, Chongqing 400716, China

\begin{abstract}
Lignin is related to not only plant disease resistance but also lodging resistance. The lignin content and six key genes ( $P A L, 4 C L, C 4 H, C C R 1, C C R 2$, and CAD) expression characteristics of the middle stem were analysed at early flowering stage and podding stage of 15 Brassica napus L. varieties with different lodging resistance level. The results indicated that the lignin content generally increased by $28 \%$ from early flowering stage to podding stage, especially by $33.5 \%$ in the varieties with stronger lodging resistance. Lignin content was significantly different between the varieties with different lodging resistance levels. The expression of key enzyme genes was remarkably different between two stages, and the expression of genes PAL, 4CL, and CCR1 was remarkably different between the varieties with different lodging resistance levels. The expression of gene $P A L$ was significantly correlated with lignin content at both stages, and the expression of gene $4 C L$ was significantly correlated with that of most other genes. Our results suggested that all the genes studied in the paper could control the lignin synthesis, and $P A L$ and $4 C L$ were the most important genes in lignin pathway.
\end{abstract}

Keywords: Lignin; qRT-PCR; Lignin pathway; Lodging resistance

木质素是植物界仅次于纤维素的第二大类植物 纤维成分, 参与植物细胞次生壁加厚、生长发育和 形态建成，影响株高、株型、生物量、抗倒伏性等 性状。此外，木质素途径还可以合成与木质素类似 的防卫型酚类物质或其他小分子物质, 参与对病等
逆境的防卫反应。目前, 在拟南芥 ${ }^{[1-3]}$ 、烟草 ${ }^{[4]}$ 、杨 树 $^{[5-7]}$ 、苜宿 ${ }^{[8]} 、$ 玉米 $^{[9]}$ 、桉树 ${ }^{[10]}$ 等植物中进行了较 为深入的研究。Bate 等 ${ }^{[11]}$ 研究表明抑制 PAL 活性会 出现非正常生长或伴随着环境适应性及抗逆性下 降。 $\mathrm{Hu}$ 等 ${ }^{[12]}$ 用反义 $\mathrm{RNA}$ 技术抑制杨树 $4 C L$ 基因表

\footnotetext{
本研究由国家自然科学基金项目(31171619)和教育部和国家外专局“作物种质资源利用创新引智基地”(B12006)资助。

*通讯作者(Corresponding author): 李加纳, E-mail: 1jn1950@swu.edu.cn, Tel: 023-68250642

第一作者联系方式: 黄杰恒, E-mail: iorihhhh@163.com; Tel: 023-68222116; 李威, E-mail: 1iwei.991ove@163.com

${ }^{* *}$ 同等贡献(Contributed equally to this work)

Received(收稿日期): 2013-01-10; Accepted(接受日期): 2013-04-22; Published online(网络出版日期): 2013-05-21.

URL: http://www.cnki.net/kcms/detail/11.1809.S.20130521.1032.001.html
} 
达，能使其活性降低 $90 \%$, 与对照相比木质素含量 也下降 45\%; 而在油菜中通过异源 $4 C L$ 基因的过量 表达, 能显著提高木质素含量, 抗病虫害及抗倒伏 能力也随之增强。Sewalt等 ${ }^{[13]}$ 研究发现, 当抑制 $\mathrm{C} 4 \mathrm{H}$ 活性降低 $80 \%$, 导致木质素含量下降 5 倍。Jones 等 ${ }^{[14]}$ 通过抑制转基因烟草 CCR 活性也能显著降低 木质素的含量, 且与模式植物拟南芥中 $C C R$ 基因的 缺陷表型一致, 会出现非正常生长, 如植株矮小、叶 片畸形、导管塌陷等现象。Halpin等 ${ }^{[15]}$ 报道，在抑制 $C A D$ 表达的转基因烟草中, 醛醇比的变化大于 $\mathrm{S} / \mathrm{G}$ 比值, 表明 CAD 活性降低, 抑制松柏醛还原相对强 烈一些，因此推测 CAD 存在不同的 CAD 同工酶。 但对于木质素的研究主要通过抑制其关键基因的表 达, 但这些关键基因的作用时期与机理的报道较 少。

油菜是我国重要的油料作物, 每年倒伏能使油 菜减产 $10 \%$ 30\%, 严重时可达 50\%以上 ${ }^{[16]}$, 同时妨 碍油菜生产的机械化。研究表明, 木质素含量与作 物抗倒伏能力呈显著正相关 ${ }^{[17-18]}$, 且在甘蓝型油菜 中茎秆木质素含量与植株的抗倒伏和抗病虫能力密 切相关，但对于木质素代谢途径本身各基因表达的 特点研究较少, 在油菜薹期和开花期, 李尧臣等 ${ }^{[19]}$ 通过对基因 $4 C L 、 F 5 H$ 和 COMT 在根、根颈和茎部 RT-PCR 结果表明 $F 5 H$ 和 $4 C L$ 基因可能是与油菜抗 倒伏相关的关键基因。张建等 ${ }^{[20]}$ 研究也认为油菜茎 秆中木质素含量差异是引起倒伏的主要因子, 抗倒 材料木质素的含量为倒伏材料的 1.2 倍。杨向东等 ${ }^{[21]}$ 对 29 个甘蓝型油菜成熟期茎段木质素含量与茎秆强 度相关性的分析发现，随着木质素含量的增加茎秆 强度亦逐渐增加。田间观察发现, 不同甘蓝型油菜 植株茎秆中部强弱与抗倒伏能力密切关联。因此, 本研究以初花期和青荚期不同倒伏性甘蓝型油菜 (Brassica napus L.) 的茎中部为材料, 分析 2 个时期 茎中部木质素含量的差异, 分析木质素代谢途径中 关键酶基因 $P A L 、 4 C L 、 C 4 H 、 C C R 1 、 C C R 2$ 和 $C A D$ 全部拷贝表达量总和, 以期发现甘蓝型油菜木质素 合成积累的关键时期及参与代谢合成的关键酶基因, 为选育抗倒伏及抗病虫品种提供依据。

\section{1 材料与方法}

\section{1 供试材料}

供试材料为重庆市油菜工程中心选育的 15 个甘 蓝型油菜品种(系), 根据多年田间观察, 将材料分为
抗倒性强、中、弱, 每种抗倒等级的材料各 5 个, 材 料来源及编号见表 1 。

表 1 试验材料名称及编号

Table 1 Names and codes of the materials

\begin{tabular}{|c|c|c|c|c|}
\hline \multirow{2}{*}{$\begin{array}{c}\text { 材料 } \\
\text { 名称 } \\
\text { Name }\end{array}$} & \multirow{2}{*}{$\begin{array}{c}\text { 抗倒强度 } \\
\text { Lodging- } \\
\text { resistance }\end{array}$} & \multirow{2}{*}{$\begin{array}{l}\text { 倒伏率 } \\
\text { Lodging } \\
\text { rate (\%) }\end{array}$} & \multicolumn{2}{|c|}{$\begin{array}{c}\text { 木质素含量 } \\
\text { Lignin content }(\%)\end{array}$} \\
\hline & & & $\begin{array}{c}\text { 初花期 } \\
\text { Flowering } \\
\text { stage }\end{array}$ & $\begin{array}{c}\text { 青荚期 } \\
\text { Podding } \\
\text { stage }\end{array}$ \\
\hline w309 & 低 Low & $\geq 80$ & 4.58 & 7.78 \\
\hline w307 & 低 Low & $\geq 80$ & 6.51 & 10.47 \\
\hline L017 & 低 Low & $\geq 80$ & 7.95 & 14.46 \\
\hline L088 & 低 Low & $\geq 80$ & 8.26 & 8.19 \\
\hline L630 & 低 Low & $\geq 80$ & 9.76 & 15.24 \\
\hline L475 & 中 Midium & $40-60$ & 10.39 & 8.95 \\
\hline L131 & 中 Midium & $40-60$ & 10.75 & 14.75 \\
\hline L031 & 中 Midium & $40-60$ & 11.31 & 15.45 \\
\hline L097 & 中 Midium & $40-60$ & 12.19 & 18.28 \\
\hline L130 & 中 Midium & $40-60$ & 12.75 & 10.84 \\
\hline L146 & 高 High & $\leq 20$ & 12.83 & 15.64 \\
\hline W356 & 高 High & $\leq 20$ & 12.94 & 14.34 \\
\hline W332 & 高 High & $\leq 20$ & 13.63 & 17.71 \\
\hline L014 & 高 High & $\leq 20$ & 13.75 & 19.93 \\
\hline L072 & 高 High & $\leq 20$ & 14.99 & 18.81 \\
\hline
\end{tabular}

\section{2 田间种植及取材}

2010 年和 2011 年在重庆市北碚区歇马镇试验 基地种植, 随机区组设计, 无重复, 每小区 3 行, 每 行 15 株。田间管理按常规生产方式进行。

参照张文华等 ${ }^{[28]}$ 对油菜抽薹期、花期和成熟期 分别测定木质素发现, 油菜木质素主要积累时期是 从花期到成熟期。因此，本试验在木质素开始大量 积累的初花期(主花序有 10 朵花以内)与最易发生倒 伏的青荚期(收获前两周)取样, 随机选取每个材料 5 株，在田间测量植株高度，从中部剪断后迅速将中 部上下各取部分装入离心管中用液氮速冻, $-70^{\circ} \mathrm{C}$ 冰 箱保存。截取中部上下各 $20 \mathrm{~cm}$ 茎秆, 烘箱中烘至 恒重, 于干燥器中保存, 用于木质素含量的测定。

\section{3 木质素测定方法}

采用 Klason 法 ${ }^{[21]}$ 测定总木质素含量。将样品用 粉碎机充分粉碎，称取 $0.3 \mathrm{~g}$ ，加入 $72 \%$ 的硫酸 7.5 $\mathrm{mL}, 30^{\circ} \mathrm{C}$ 消化 $1 \mathrm{~h}$ 后, 用蒸馏水稀释至硫酸浓度为 $4 \%, 121^{\circ} \mathrm{C}$ 下处理 $1 \mathrm{~h}$, 冷却至室温后, 单层滤纸过 滤, 最后将残渣烘干称重。木质素含量 $=$ (木质素质 量/所取材料的质量) $\times 100 \%, 3$ 次重复, 取平均值。 
1.4 木质素合成相关基因表达分析

1.4.1 总 RNA 的提取和 cDNA 的合成 用试剂 盒(TIANGEN BIOTECH)提取总 RNA。用 DNase I (TaKaRa)除去总 RNA 中混有的少量 DNA, Thermo NanoDrop 2000c 微量紫外分光光度计测定 RNA 浓 度并用 $1.2 \%$ 的琼脂糖凝胶电泳检测总 RNA 的完整 性。按照说明书合成总 $\mathrm{cDNA}$ 第一链, $20 \mu \mathrm{L}$ 体系含 $1 \mu \mathrm{L}$ 总 RNA、 $50 \mu \mathrm{mol} \mathrm{L}^{-1}$ Oligo(dT) 18 混液于 $70^{\circ} \mathrm{C}$ 水浴 $10 \mathrm{~min}$; 然后加入 $200 \mathrm{U}$ 反转录酶、 $10 \mathrm{mmol} \mathrm{L}^{-1}$ dNTPs、40 U RNase 抑制剂(TaKaRa)及灭菌的双蒸 水, 置 $42^{\circ} \mathrm{C}$ 水浴 $15 \mathrm{~min}$, 最后于 $85^{\circ} \mathrm{C}$ 变性 $5 \mathrm{~s}$, 冰上 冷却, $-20^{\circ} \mathrm{C}$ 保存备用。

1.4.2 qRT-PCR 分析 利用已公布的拟南芥基因 组序列(TAIR 数据库, http://www.arabidopsis.org/)及 白菜基因组序列(BRAD 数据库, http://brassicadb.org/) 和已报道的油菜木质素合成相关的关键基因序列, 在 NCBI 中进行多重比对, 用软件 Geneious 4.8.5 设 计引物(表 2)。实时苂光定量 PCR 反应体系 $20 \mu \mathrm{L}$ 含 $2 \times$ SYBR Premix Ex Taq (TaKaRa) $10 \mu \mathrm{L}$ 、 ROX Reference Dye II $0.4 \mu \mathrm{L}$ 、cDNA $2 \mu \mathrm{L}$ 、Primer-F 0.4 $\mu \mathrm{L}$ 、Primer-R $0.4 \mu \mathrm{L}$, 补水至总体积 $20 \mu \mathrm{L}$ 。扩增反 应程序为 $95^{\circ} \mathrm{C} 30 \mathrm{~s}, 95^{\circ} \mathrm{C} 5 \mathrm{~s}, 58^{\circ} \mathrm{C} 34 \mathrm{~s}, 72^{\circ} \mathrm{C} 30 \mathrm{~s}, 40$ 个循环(退火温度根据不同引物进行调整), 采用 $2^{-\Delta \Delta \mathrm{CT}}$ 法分析数据, 确定基因的相对表达量。每个取 样点设 3 次技术重复, 试验共设 3 次生物学重复, 分
别测定所选 15 份材料 2 个时期关键酶基因的全部拷 贝表达量总和。

\section{2 结果与分析}

\section{1 木质素含量分析}

2.1.1 初花期木质素含量 初花期木质素含量在 $4.58 \%$ 14.99\%间, 平均为 $10.85 \%$ 。其中抗倒性较弱、 抗倒性一般及抗倒性较强材料的木质素含量平均为 $7.41 \% 、 11.48 \%$ 和 $13.63 \%$, 抗倒性较强比较弱材料木 质素含量高 6.22 个百分点, 各抗倒等级间木质素含 量差异极显著(表 1)。

\subsection{2 青英期木质素含量 青荚期木质素含量在} $7.78 \%$ 19.93\%间, 平均值为 $13.9 \%$ 。其中抗倒性较 弱、抗倒性一般及抗倒性较强材料的木质素含量平 均为 $8.84 \% 、 15.13 \%$ 和 $18.19 \%$ 。抗倒性较强比较弱 材料木质素含量高 9.35 个百分点(表 1)。与初花期相 比, 青荚期的茎秆木质素含量平均高 3.04 个百分点, 增加了 $28 \%$, 不同抗倒类型材料的平均木质素含量 均有明显增加, 但抗倒性强的材料木质素含量增加 最明显, 增幅达到 $33.5 \%$ 。3 组不同抗倒性材料在 2 个时期间木质素含量方差分析见表 3 。

\section{2 木质素合成相关的关键基因 qRT-PCR 表达} 分析

从图 1 看出, 基因 $P A L 、 4 C L 、 C C R 1 、 C C R 2$ 和 $C A D$ 的全部拷贝表达量总和从初花期到青荚期明显

表 2 本研究中所用到的引物及其扩增产物

Table 2 Primer sequences and amplification products size

\begin{tabular}{|c|c|c|c|c|}
\hline \multirow{2}{*}{$\begin{array}{l}\text { 基因 } \\
\text { Gene }\end{array}$} & \multicolumn{2}{|c|}{ 引物序列 Primer sequence (5'-3') } & \multirow{2}{*}{$\begin{array}{c}\text { PCR 产物长度 } \\
\text { Amplicon size (bp) }\end{array}$} & \multirow{2}{*}{$\begin{array}{c}\text { 退火温度 } \\
T_{\mathrm{m}}\left({ }^{\circ} \mathrm{C}\right)\end{array}$} \\
\hline & Forward & Reverse & & \\
\hline$P A L$ & GCAGCGCCACGTACCCGTTGAT & AGCACCGCCTTGAGCTCCTCCT & 142 & 61.86 \\
\hline $\mathrm{C} 4 \mathrm{H}$ & GCTCGCTGGCTATGACATCCCA & ACGTCCAACACCAAACGGCAAG & 181 & 60.93 \\
\hline $4 C L$ & GACGACGTGGTGGCGCTTCCTT & ATCGCCGTCGACTTGCTGAGCG & 111 & 61.86 \\
\hline CCR1 & CCGAACCGTGACCCTGAAGCCG & CGGCTTGCTCCGCCACCATCTT & 112 & 61.92 \\
\hline CCR2 & CGGCGGTGAACGGAGCCAAGTT & TGACCGCTGACTGGAGCGGTGG & 297 & 62.11 \\
\hline$C A D$ & GTGCTTGAGTTGGGCTGCACGA & CAACACCAACGTGGTCTCCGAC & 254 & 59.75 \\
\hline
\end{tabular}

表 3 初花期和青荚期木质素含量方差分析

Table 3 Variance analysis of lignin content in early flowering stage and podding stage

\begin{tabular}{|c|c|c|c|c|c|}
\hline $\begin{array}{c}\text { 变异来源 } \\
\text { Variation source }\end{array}$ & $\begin{array}{c}\text { 平方和 } \\
S S \\
\end{array}$ & $\begin{array}{c}\text { 自由度 } \\
d f\end{array}$ & $\begin{array}{c}\text { 均方 } \\
M S\end{array}$ & $\begin{array}{c}F \text { 值 } \\
F \text {-value }\end{array}$ & $\begin{array}{c}P \text { 值 } \\
P \text {-value }\end{array}$ \\
\hline 时期间 Stages & 0.0078 & 1 & 0.0078 & 52.1804 & $1.83 \mathrm{E}-07$ \\
\hline 类别间 Categories & 0.0314 & 2 & 0.0157 & 105.5231 & $1.28 \mathrm{E}-12$ \\
\hline 互作 Interaction & 0.0013 & 2 & 0.0006 & 4.3654 & 0.0242 \\
\hline 误差 Error & 0.0036 & 24 & 0.0001 & & \\
\hline 总变异 Variance & 0.0440 & 29 & & & \\
\hline
\end{tabular}



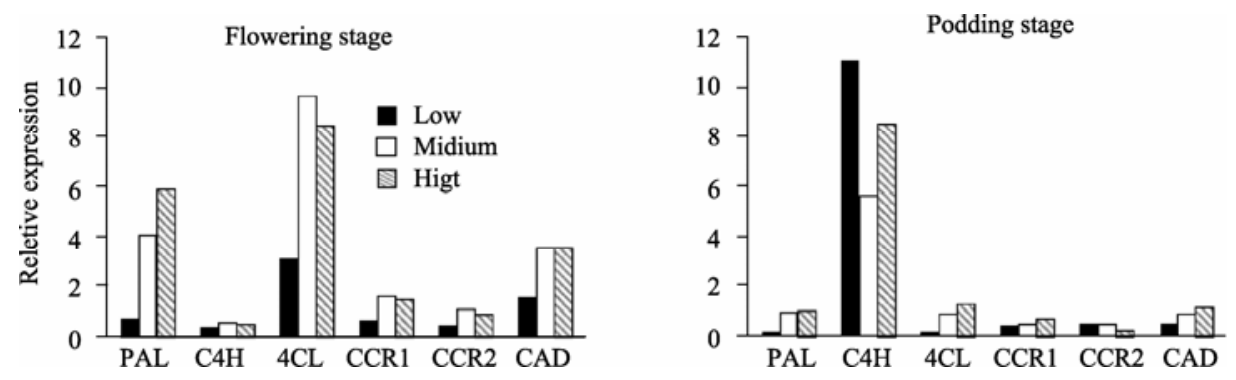

图 1 不同抗倒性材料间各木质素关键基因全部拷贝表达量总和

Fig. 1 Key enzyme genes expression in different lodging-resistant materials at early flowering stage and podding stage

下降，平均下降 $80 \% 、 88 \% 、 61 \% 、 53 \%$ 和 $71 \%$, 而 $\mathrm{C} 4 \mathrm{H}$ 基因在青英期的表达量较初花期平均上升 15 倍。初花期各基因表达量差异极显著, 各抗倒性材 料间表达量差异也极显著(表 4)。各基因表达量与 木质素含量均正相关, 其中 PAL 与木质素含量显 著相关, 抗倒性强组表达量是抗倒性弱组的 7.67 倍, 其次是基因 $C 4 H$ 和 $C C R 1$, 其中基因 $4 C L$ 表达 量与 $P A L 、 C C R 1 、 C C R 2$ 和 $C A D$ 基因表达量极显
著相关(表6); 青荚期各基因表达量差异极显著, 但 各抗倒性材料间表达量差异不显著(表 4), 基因 $P A L 、 4 C L$ 表达量与木质素含量极显著相关, 抗倒 性强组表达量分别是抗倒性弱组的 5.26 倍和 7.67 倍, 且 2 个时期间表达量差异显著(表5)而基因 $C 4 \mathrm{H}$ 和 CCR2 与木质素含量呈负相关, 其中基因 $4 C L$ 表 达量与 PAL 和 CAD 基因表达量均极显著相关 (表 $6)$ 。

表 4 初花期(FS)和青荚期(PS)关键基因表达量的方差分析

Table 4 Variance analysis of genes expression in early flowering stage (FS) and podding stage (PS)

\begin{tabular}{|c|c|c|c|c|c|c|c|c|c|}
\hline \multirow{2}{*}{$\begin{array}{c}\text { 变异来源 } \\
\text { Variation source }\end{array}$} & \multicolumn{2}{|c|}{ 平方和 $S S$} & \multirow{2}{*}{$\begin{array}{c}\text { 自由度 } \\
d f\end{array}$} & \multicolumn{2}{|c|}{ 均方 $M S$} & \multicolumn{2}{|c|}{$F$ 值 $F$-value } & \multicolumn{2}{|c|}{$P$ 值 $P$-value } \\
\hline & FS & PS & & FS & PS & FS & PS & FS & PS \\
\hline 基因间 Intergenic & 548.1042 & 792.5813 & 6 & 91.3507 & 132.0969 & 28.4710 & 14.403 & 0.0001 & 0.0001 \\
\hline 类别间 Categories & 92.7209 & 6.2345 & 2 & 46.3604 & 3.1172 & 14.4490 & 0.340 & 0.0001 & 0.7128 \\
\hline 互作 Interaction & 116.2707 & 73.8306 & 12 & 9.6892 & 6.1525 & 3.0200 & 0.671 & 0.0015 & 0.7745 \\
\hline 误差 Error & 269.5165 & 770.4293 & 84 & 3.2085 & 9.1718 & & & & \\
\hline 总变异 Variance & 1026.6122 & 1643.0756 & 104 & & & & & & \\
\hline
\end{tabular}

表 5 两个时期各基因表达量方差分析(用 $\boldsymbol{F}$ 值加*表示)

Table 5 Variance analysis of each key enzyme gene expression during the early flowering stage and podding stage

\begin{tabular}{cccccc}
\hline \multirow{2}{*}{$\begin{array}{c}\text { 变异来源 } \\
\text { Variation source }\end{array}$} & \multicolumn{4}{c}{ 基因 Gene } \\
\cline { 2 - 6 } & $P A L$ & $C 4 H$ & $4 C L$ & CCR1 & CCR2 \\
\hline 生育期间 Stages & $18.4756^{* *}$ & $14.7401^{* *}$ & $49.9115^{* *}$ & $22.0726^{* *}$ & 4.6588 \\
类别间 Categories & $6.9078^{* *}$ & 0.5484 & $6.4644^{* *}$ & $4.7319^{*}$ & 0.7475 \\
互作 Interaction & $3.5222^{*}$ & 0.6219 & $3.8018^{*}$ & 2.8349 & 3.0038 \\
\hline
\end{tabular}

${ }^{*}$ 和 ${ }^{* *}$ 表示在 $5 \%$ 和 $1 \%$ 水平上差异显著。 ${ }^{*}$ and ${ }^{* *}$ denote significant difference at $5 \%$ and $1 \%$ probability levels, respectively.

表 6 初花期(FS)和青莱期(PS)关键酶基因表达量相关系数

Table 6 Correlation coefficients of expression between key enzyme genes at the early flowering stage (FS) and podding stage (PS)

\begin{tabular}{|c|c|c|c|c|c|c|c|c|c|c|c|c|}
\hline \multirow{2}{*}{$\begin{array}{l}\text { 基因 } \\
\text { Gene }\end{array}$} & \multicolumn{2}{|c|}{$\mathrm{LC}$} & \multicolumn{2}{|c|}{ PAL } & \multicolumn{2}{|c|}{$4 \mathrm{CL}$} & \multicolumn{2}{|c|}{$\mathrm{C} 4 \mathrm{H}$} & \multicolumn{2}{|c|}{ CCR1 } & \multicolumn{2}{|c|}{ CCR2 } \\
\hline & FS & PS & FS & PS & FS & PS & FS & PS & FS & PS & FS & PS \\
\hline$P A L$ & $0.58^{*}$ & $0.61^{* *}$ & & & & & & & & & & \\
\hline $4 C L$ & 0.43 & $0.73^{* *}$ & $0.73^{* *}$ & $0.85^{* *}$ & & & & & & & & \\
\hline $\mathrm{C} 4 \mathrm{H}$ & 0.28 & -0.29 & 0.12 & 0.14 & -0.21 & 0.05 & & & & & & \\
\hline CCR1 & 0.44 & 0.28 & $0.67^{* *}$ & -0.10 & $0.98^{* *}$ & 0.17 & -0.19 & 0.11 & & & & \\
\hline CCR2 & 0.39 & -0.19 & 0.29 & -0.14 & $0.65^{* *}$ & -0.18 & -0.22 & 0.18 & $0.67^{* *}$ & -0.28 & & \\
\hline$C A D$ & 0.44 & 0.43 & 0.43 & $0.56^{*}$ & $0.70^{* *}$ & $0.69^{* *}$ & 0.10 & 0.33 & $0.74^{* *}$ & 0.20 & $0.65^{* *}$ & 0.06 \\
\hline
\end{tabular}

${ }^{*}$ 和 ${ }^{* *}$ 表示在 $5 \%$ 和 $1 \%$ 水平上差异显著。 ${ }^{*}$ and ${ }^{* *}$ denote significant difference at $5 \%$ and $1 \%$ probability levels, respectively. 


\section{3 讨论}

油菜抗病、抗逆和抗倒性弱是限制我国油菜生 产面积和机械化生产的主要因素, 提高油菜茎秆木 质素含量可以显著提高油菜抗倒性, 但关于茎秆木 质素代谢的生理和分子生物学研究还很薄弱。前人 对于甘蓝型油菜木质素的研究也主要集中在油菜成 熟后期的根部以上茎秆 ${ }^{[19-22]}$, 而对于甘蓝型油菜最 易发生折断的茎秆中部以及最易发生倒伏的青荚期 的研究报道甚少。本研究表明, 在 2 个时期各材料的 木质素含量均差异极显著, 初花期到青荚期油菜茎 秆木质素含量平均增加了 $28.0 \%$, 抗倒性强的材料 木质素增加最明显, 达到 $33.5 \%$, 其中上升幅度最 大的材料是 L17, 平均增加 $45.0 \%$; 但材料 L088、

L130 和 L475 的木质素含量却略有下降, 这可能是 这些材料在青荚期的木质素累计较少, 加上抽样误 差(因为是花期和青荚期是不同的单株)所致。在初花 期, 木质素代谢途径各主要基因的全部拷贝总表达 量与木质素含量正相关, 说明初花期可能是油菜茎 秆中部木质素积累的关键时期。另外, 木质素含量 与田间观察的抗倒性具有明显的正相关, 与前人的 研究结果一致 ${ }^{[19-22]}$ 。

虽然研究者们对木质素的合成已经进行了深入 研究, 主要的关键酶基因和调控基因在拟南芥、烟 草、金鱼草、杨树、欧芹、桉树、苜宿、水稻、葡 萄等众多植物中被克隆, 但木质素途径比较复杂, 表现在中间产物及木质素的侧基修饰类型丰富, 合 成途径呈网络状, 许多产物的生成具有不止一条的 合成途径 ${ }^{[23]}$, 且这些基因均表现为多基因家族 ${ }^{[24-25]}$, 甘蓝型油菜木质素合成代谢作用机制尚不清楚。本 研究根据已报道的研究成果选取了木质素代谢途径 中 6 个比较重要的功能基因, 经 qRT-PCR 分析表明, 木质素合成的下游特异途径所涉及的酶 CCR 和 $C A D$ 基因在青荚期明显下调, $C C R 1$ 和 $C C R 2$ 分别平 均下调 $60 \%$ 和 $50 \%$, 而 $C A D$ 平均下调 $71 \%$, 说明 $C C R$ 和 $C A D$ 基因主要参与木质素的合成, 随着青荚 期茎秆木质素合成量的减少，它们的表达量也明显 降低。作为木质素合成途径的上游基因, $C 4 H$ 的表达 量在青荚期分别平均上调 15 倍, 推测是由于 $\mathrm{C} 4 \mathrm{H}$ 酶 在苯丙烷途径中主要催化苯丙氨酸到羟基肉桂酸及 其辅酶 A 酯类的过程, 这些产物除用于木质素的生 物合成外, 更多的还是很多酚类化合物的前体, 说 明在油菜青荚期茎秆中, 类黄酮代谢还比较旺盛。

研究结果还发现, 作为公共苯丙烷途径(木质素
与色素的共享途径)第一个关键酶基因 $P A L$ 的全部拷 贝总表达量在青荚期大幅度下调, 高达 $79 \%$, 这与 $\mathrm{C} 4 \mathrm{H}$ 基因的行为相悖, 这种现象是否是因为在油菜 花期至青荚期前期茎秆中 $P A L$ 表达量高, 已合成了 足够的肉桂酸中间产物, 青荚期后期已不需要该基 因的过多参与? 还需要进一步的试验证明。不过, 无论在初花期还是青荚期, 基因 $P A L$ 的表达量与茎 秆木质素含量均呈极显著正相关, 还是说明该基因 与茎秆木质素的关系密切。

本研究中, 其他基因全部拷贝总表达量与木质 素含量相关性不显著, 鉴于这些基因都是木质素代 谢途径中的必需基因, 说明油菜茎秆木质素合成代 谢途径可能受这些基因的共同作用，而非单个关键 基因的影响。在青荚期, 基因 CCR1、CCR2 和 $C A D$ 表达量显著下降, 此时木质素含量却没有明显变化, 说明木质素的代谢积累已处于基本停滞。

从基因间全部拷贝总表达量的相关性来看, 初 花期基因 4CL 表达量与 $P A L 、 C C R 1 、 C C R 2$ 和 $C A D$ 基因表达量极显著相关, 青荚期 $4 C L$ 表达量与 $P A L$ 和 $C A D$ 表达量极显著相关, 说明 $4 C L$ 是关联苯丙烷 代谢途径、类黄酮代谢途径和木质素代谢途径的重 要基因 ${ }^{[26]}$, 是木质素代谢中承前启后的关键酶基因, 这与杨向东等 ${ }^{[21]}$ 结论相吻合。在初花期, CCR 家族 中的 CCR1 基因在高抗倒性材料中的表达量为低抗 倒性材料的 2.36 倍, 而 CCR2 基因表达量在不同抗 倒伏材料中差异并不明显, 说明 CCR1 主要参与茎 秆的木质化过程。另外, Buahcer 等 ${ }^{[27]}$ 研究表明抑制 $\mathrm{CAD}$ 酶活性时, 植物木质素含量并未明显改变, 仍 维持在正常的水平, 但相应的肉桂醇含量减少。 Halpin 等 ${ }^{[15]}$ 则认为抑制 $C A D$ 表达的转基因烟草中, 醛醇比的变化大于 $\mathrm{S} / \mathrm{G}$ 比值, 表明 $\mathrm{CAD}$ 活性降低, 抑制松柏醛还原相对强烈, 推测 CAD 存在同工酶, 即 $\mathrm{SAD}$ 。但本文的研究发现初花期 $C A D$ 基因在高抗 倒组是低抗倒组表达量的 2.28 倍, 并且进入青荚期 后显著下调, 幅度达到 $71 \%$, 说明 $C A D$ 在木质素的 合成代谢和调控中起到了重要作用。

研究木质素途径中几个重要关键基因在油菜生 育后期茎秆中的表达特点基因间的相互关联作用, 对于深入研究甘蓝型油菜的茎秆木质素合成代谢机 制具有重要意义。

\section{4 结论}

初花期是油菜茎秆中部木质素合成的重要时期; 茎秆中部木质素含量与抗倒伏性有显著的相关性; 
不同遗传背景材料间木质素代谢并非由某个功能基 因所控制，而是多个基因相互协调作用的结果，基 因 $P A L$ 的表达与木质素含量相关性最强, 4CL 基因 的表达量与其他基因表达量相关性最强, 而 $C 4 H$ 基 因表达量与木质素含量及各基因表达量相关性最 弱。推测调控 $P A L$ 和 $4 C L$ 基因对油菜茎秆木质素含 量的影响会更明显。

\section{References}

[1] Franker R, Mcmichael C M, Meyer K, Shirley A M, Cusumano J C, Chapple C. Modified lignin in tobacco and poplar plants over-expressing the Arabidopsis gene encoding ferulate-5hydroxylase. Plant J, 2000, 22: 223-234

[2] Soltani B M, Ehlting J, Douqlas C J. Genetic analysis and epigenetic silencing of At4CL1 and At4CL2 expression in transgenic Arabidopsis. Biotechnology, 2006, 1: 1124-1136

[3] Sibout R, Baucher M, Gatineau M, Doorsselaere J V, Mila I, Pollet B, Maba B, Pilate G, Lapierre C, Boerjan W, Jouanin L. Expression of a poplar cDNA encoding a ferulate-5-hydroxylase/ coniferaldehyde5-hydroxylase increases S lignin deposition in Arabidopsis thaliana, Plant Physiol Biochem, 2002, 40: 1087-1096

[4] Zhong R W. Morrison W H III, Negrel J, Ye Z H. Dual methylation pathway in lignin biosynthesis. Plant Cell, 1998, 10: 2033-2045

[5] Zhong R W. Morrison W H III, Himmelsbach D S, Farris L, Poole II, Ye Z H. Essential role of cafeoylcoenzyme o-methy 1 transferase in lignin biosynthesis in woody poplar plants. Plant Physiol, 2000, 124: 563-578

[6] Hu W J, Harding S A, Lung J, Popko J L, Ralph J, Stokke D D, Tsai C J, Chiang V L. Repression of lignin biosynthesis promotes cellulose accumulation and growth in transgenic trees. Nat Biotechnol, 1999, 17: 808-812

[7] Zhao Y L, Lu H, Tao X J, Chen X M, Jiang X N. Modulate the lignin biosynthesis by expression GRP1.8 promoter: anti-4CL1 gene in transgenic tobacco. J Beijing For Univ, 2003, 25(4): 16-20

[8] Guo D J, Chen F, Inoue K, Blount K J, Dixon R A. Down-regulation of caffeic acid 3-o-methyl transferase in transgenic alfalfa: impacts on lignin structure and implications for the biosynthesis of G and S lignin. Plant Cell, 2001, 13: 73-88

[9] Piquemal J, Chamayou S, Nadaud I, Beckert M, Barrière Y, Mila I, Lapierre C, Rigau J, Puigdomenech P, Jauneau A, Digonnet C, Boudet A M, Goffner D, Pichon M, Down-regulation of caffeic acid o-methyl transferase in maize revisited using a transgenic approach. Plant Physiol, 2002, 130: 1675-1685

[10] Thumma B R, Nolan M F, Evans R, Moran G F. Polymorphisms in cinnamoyl CoA reductase $(C C R)$ are associated with variation in microfibril angle in Eucalyptus spp. Genetics, 2005, 171: $1257-1265$

[11] Bate N J, Orr J, Ni W, Meromi A, Nadler-Hassar T, Doerner P W, Dixon R A, Lamb C J, Elkind Y. Quantitative relationship between phenylalanine ammonialyase levels and phenylpropanoid accumulation in transgenic tobacco identifies a rate-determining step in natural product synthesis. Proc Natl Acad Sci USA, 1994, 91: 7608-7612

[12] Hu W J, Akiyoshi K, Tsaich J. Compartmentalized expression of two structurally and functionally distinct 4-coumarate: CoA ligase genes in aspen (Populus tremuloides). Proc Natl Acad Sci
USA, 1998, 95: 5407-5412

[13] Sewalt V J H, Ni W, Jung H G, Dixon R A. Lignin impact on fiber degradation: increased enzymatic digestibility of genetically engineered tobacco (Nicotiana tabacum) stems reduced in lignin content. J Agric Food Chem, 1997, 45: 1977-1983

[14] Jones L, Ennos A R, Turner S R. Cloning and characterization of irregular xylem4 (irx4): a severely lignin-deficient mutant of Arabidopsis. Plant J, 2001, 26: 205-216

[15] Halpin C, Knight M E, Foxon G A. Manipulation of lignin quality by down-regulation of cinnamyl alcohol dehydrogenase. Plant J, 1994, 6: 339-350

[16] Jiang W-M(姜维梅), Zhang D-Q(张冬青), Xu C-X(徐春霄). Studies on the stem anatomy of Brsssica oil to lodging. $J$ Zhejiang Agric Univ (Agric Life Sci)(浙江大学学报·农业与生命科学版), 2001, 27(4): 439-442 (in Chinese with English abstract)

[17] Thompson D L. Stalk strength of corn as measured by crushing strength and rind thickness. Crop Sci, 1963, 3: 323-329

[18] Zuber M S, Grogan C O. A new technique for measuring stalk strength in corn. Crop Sci, 1961, 1: 378-380

[19] Li X-C(李尧臣), Qi C-K(戚存扣). Lignin content and key gene expression in lignin synthesis of Brassica napus L. with lodging resistance. Jiangsu J Agricl Sci (江苏农业学报), 2011, 27(3): 481-48 (in Chinese with English abstract)

[20] Zhang J(张建), Chen J-C(陈金城), Tang Z-L(唐章林), Wang $\mathrm{R}$ (王瑞). Study on the physic-chemical properties of stem as related to lodging in rape. J Southwest Agric Univ (西南农业大学 学报), 2006, 28(5): 763-765 (in Chinese with English abstract)

[21] Yang X-D(杨向东). The Study on the Relationship between Lignin Biosynthsis Manipulation and Brassica napus Resistance to Sclerotinia sclerotiorum and Lodging. PhD Dissertation of Chinese Academy of Agricultural Sciences, 2006 (in Chinese with English abstract)

[22] Yang X-D(杨向东), Wang H-Z(王汉中), Hu Z-M(胡赞民), Liu G-H(刘贵华), Shen J-X(沈金雄). The cloning, expression and activity assays of 4-CL cDNA from the Populus tomentosa. $J$ Huazhong Agric Univ (华中农业大学学报)，2006，25(2): 101-105 (in Chinese with English abstract)

[23] Yamauchi K, Yasuda S, Hamada K, Tsutsumi Y, Fukushima K. Multiform biosynthetic pathway of syringyl lignin in angiosperms. Planta, 2003, 216: 496-501

[24] Bugos R, Chiang V, Campbell W. cDNA cloning, sequence analysis and seasonal expression of lignin-bispecific caffeic acid/5-hydroxyferulic acid O-methyl transferase of aspen. Plant Mol Biol, 1991, 17: 1203-1215

[25] Tamagnone L, Merida A, Parr A, Mackay S, Culianez-Macia FA, Roberts K, Martin C. The AmMYB308 and AmMYB330 transcription factors from antirrhinum regulate phenylpropanoid and lignin biosynthesis in transgenic tobacco. Plant Cell, 1998, 10: $135-154$

[26] Vanholme R, Storme V, Vanholme B, Sundin L, Christensen J H, Goeminne G, Halpin C, Rohde A, Morreel K, Boerjan W. A systems biology view of responses to lignin biosynthesis perturbations in Arabidopsis. Plant Cell, 2012, 24: 3506-3529

[27] Baucher M, Monties B, Van Montage M, Boerjan W. Biosynthesis and genetic engineer in lignin. Crit Rev Plant Sci, 1998, 17: $125-197$

[28] Zhang W-H(张文华). Genetic Analysis and QTL Mapping of Lodging Resistance Related Traits in Brassica napus. MS Thesis of Huazhong Agricultural University, 2010 (in Chinese with English abstract) 